\title{
Surgery for cervical disc disease. Why go for the worst solution?
}

\author{
Bernhard Meyer
}

Received: 18 March 2010 /Accepted: 23 March 2010/Published online: 11 April 2010

(C) Springer-Verlag 2010

The agenda of these authors was to verify — or rather eraseconcerns about the negative long-term effects of anterior cervical discectomy (ACD) without interposed graft, spacer or prosthesis. This is, by the way, the correct term for this procedure, because fusion is exactly what you thrive for. Just because "ACD without fusion" is a common misnomer, it does not get better by steady repetition.

Their conclusion is that this is the cheapest, simplest and fastest method and their preference especially for a singlelevel soft disc, because they think that the clinical long-term outcome of ACDF and ACD is the same.

The reason to favour publication of this paper [1] is not because I share the authors' views or support their conclusions. The contrary is true. I think the importance of this communication lies in the fact that it provides the opportunity to point out some common misconceptions.

On a more general level, these are:

1. People tend to believe that a study with an inappropriate design produces better answers after a longer follow-up.

2. The same people tend to confuse statistical significance and clinical relevance. Just because statistics do not prove a difference, the inference that there is none in reality is not true.

Many methodological limitations prohibit any firm conclusions here. I will name a few. A retrospective analysis of a selected surgical series over such a long time can, per definition, never give an answer to these questions. It is not consecutive, because other patients during this

\section{B. Meyer $(\bowtie)$}

Department of Neurosurgery, Technical University of Munich,

Klinikum rechts der Isar, Ismaninger Str. 22,

81675 Munich, Germany

e-mail: Bernhard.meyer@1rz.tum.de period were treated differently at this institution. According to their own reference 45 , the authors have obviously treated 100 patients with cages in the same time span. (Why is that so, if ACD without cage is the best?)

Furthermore, the authors imply that they have a $100 \%$ follow-up rate over 11 years in a retrospective study, something I have never seen before. Are the authors sure that none of their patients receiving an ACD was lost to follow-up? Because this would again introduce an important element of selection bias and could also explain an unusually high fusion $(90 \%)$ and satisfaction $(96 \%)$ rate. On the other hand, only 90 of 125 patients were eligible for assessment of fusion and the clinical outcome measure was crude, since "poor" was simply defined as being worse than before surgery. This alone may be sufficient to explain these numbers.

However, the above is less important than the question on how to judge the literature and the available evidence.

Conflicting or unequivocal results of several studies on the same topic call for a critical appraisal and not just the inference that this is the equivalent of a proof of non-inferiority.

The evidence needs then to be sorted by its quality, which gives a slightly different picture according to my interpretation. The better quality studies rather point into the direction that ACD produces somewhat less favourable results.

However, I concede that there is no unequivocal proof of its inferiority, as it is the case with all surgical methods available for the treatment of cervical degenerative disease. Another example is the debate of cages versus arthroplasty. Why is that so? The answer is easy. Because all methods are very successful, only small differences exist, which are very hard to prove by statistical means. (Again, lack of statistical significance does not imply there is no clinically meaningful difference.) 
Therefore, I completely concur with the authors' notion that the decision to use this method lies at the individual surgeon's discretion and he or she can certainly not be held responsible legally or otherwise for its use.

However, I ask myself if this is the smartest way to deal with such kind of a problem and would like to propose a different strategy:

If you do not have "statistics" or "EBM" to prove something, then why not use common sense? Almost everybody will agree that ACD without an interposing element is the most destructive procedure you can choose among the available ones. This is evidenced by (1) the high rate of kyphotic deformities on the long run, which nobody calls into question and (2) the higher incidence of postoperative pain, which most people will accept as a given and well proven.

Accepting this, you should ask yourself if you have virtually no doubts at all that this may produce a clinically meaningful problem in some of your patients down the line. Because if you had a single doubt, then you would need good arguments in favour of this destructive approach, which leaves a void initially and will disrupt a motion segment later on.

Money is certainly not a good one. A patient with a single-level radiculopathy due to a soft disc can very well be treated with a posterior foraminotomy. It is as quick, as easy and as cheap as an ACD plus having the advantage of leaving the motion segment intact. Most studies will show no inferiority for this strategy with regard to clinical outcome.

Even if you think you must choose an anterior approach, time and money are nowadays not really valid arguments against any form of interposing element, which will at least secure sagittal alignment in a higher proportion of your patients. Cages without filling have very low implant costs in most countries and are adequately reimbursed. An experienced surgeon can insert them within a few minutes.

Does anyone really think this justifies nowadays the plea for a conceptually outdated and non-logical procedure? I certainly do not!

I can accept that money is an issue with arthroplasty, because it is so much more expensive than a simple cagebut this is also no reason to go that far back in time. It may well be that some "younger" surgeons use instrumentation and implants in a very liberal and enthusiastic manner. However, some more enthusiasm would sometimes suit the wiser ones, too.

\section{Reference}

1. Palma L, Mariottini A, Carangelo B, Muzii VF, Zalaffi A (2010) Favourable long-term clinical outcome after anterior cervical discectomy. A study on a series of 125 patients undergoing surgery a mean of 11 years earlier. Acta Neurochir. doi:10.1007/s00701-010-0650-5 